\title{
Un exemple de conversion d'une table de production en volume en tables de production en biomasse : le chêne dans le secteur ligérien
}

\author{
J.L. BISCH * \\ INRA, Station de Sylviculture, Centre de Recherches d'Orléans \\ Ardon, F 45160 Olivet
}

\begin{abstract}
Résumé
La table de production du chêne sessile dans le secteur ligérien, proposée par PARDÉ en 1962 , est convertie en quatre tables de production en biomasse correspondant chacune à une partie de l'arbre ou à l'arbre entier, biomasse foliaire exclue.

La conversion est réalisée par l'intermédiaire de tarifs de la forme Biomasse $=a+b$ VBF $(\mathrm{VBF}=$ volume bois fort $)$, établis avec un échantillon de 18 arbres convenablement choisis.

Ces tarifs sont appliqués au volume bois fort de larbre moyen enlevé en éclaircie ou restant dans le peuplement après éclaircie.

Nous calculons une erreur statistique théorique due au modèle sur l'estimation de la biomasse disponible sur pied, la biomasse enlevée à chaque éclaircie et la production totale.

Ainsi, la production moyenne maximale, au seuil de confiance de 95 p. 100, est évaluée à $3,91 \pm 0,24$ tonnes/ha/an.

Nous donnons une relation très simple entre la table 1 (biomasse totale) et la table 2 (biomasse du houppier).

La précision et l'intérêt des différentes tables sont discutés.

Mots clés : Biomasse, table de production, Quercus petraea liebl., futaie, régression, erreur
\end{abstract}

\section{Introduction}

Voici maintenant plus de dix ans que la perspective d'une raréfaction des stocks énergétiques classiques a provoqué un regain d'intérêt pour le bois en tant que source d'énergie renouvelable.

L'idée d'une utilisation complète de l'arbre entier (Young, 1974 ; PArdé, 1977) a fait son chemin et de nombreux travaux ont permis l'estimation de la biomasse disponible dans les peuplements forestiers.

(*) Adresse actuelle : Office National des Forêts, Division de Mulhouse, 21, rue de l'Est, 68100 Mulhouse. 
L'ouvrage de Cannell. (1982) réunit la plus grande partie des estimations réalisées à travers le monde avant cette date.

Un récent rapport de l'O.C.D.E. (1984) présente une estimation du potentiel énergétique que constituent les résidus de l'exploitation et de la transformation du bois dans différents pays. Les travaux que nous présentons ici sont une contribution supplémentaire à cette estimation.

Une étude sur la répartition de la biomasse dans le chêne du secteur ligérien traité en futaie ou en taillis sous futaie nous a permis d'établir des relations linéaires entre la biomasse de certaines parties de l'arbre et son volume bois fort total (BIsch et AUCLAIR, 1987).

La possibilité nous était alors offerte de convertir la table de production en volume proposée par PARdé (1962) en différentes tables de production en biomasse.

Nous avons choisi de construire quatre tables dont les intérêts sont différents :

- une table de production en biomasse aérienne totale dont l'intérêt est surtout fondamental ; elle permet la comparaison avec les productions des autres traitements sylvicoles et notamment du taillis ;

- une table de production en biomasse du houppier;

- une table de production en biomasse des bois de diamètre inférieur à $7 \mathrm{~cm}$;

- une table de production en biomasse des bois de diamètre inférieur à $4 \mathrm{~cm}$.

Ces trois dernières ont un intérêt pratique, elles permettent à chaque passage en éclaircie d'estimer la biomasse disponible en petits bois ou bois de houppier.

Aucune de ces tables ne prend en compte la biomasse foliaire.

\section{Matériel et methode}

- Les arbres que nous avons abattus et mesurés proviennent de différentes placettes appartenant aux «couples futaie/taillis sous futaie " installés en région Centre pour les travaux de LE GofF (1984).

L'échantillon de 18 tiges de futaie a été conçu pour obtenir 3 arbres dans 6 classes d'âge définies entre 40 et 140 ans. Il tient aussi compte des variables circonférence à $1,30 \mathrm{~m}(\mathrm{C} 130)$, hauteur totale (HT), hauteur relative du houppier (HR) et indice de productivité de la station. La hauteur relative du houppier est définie par le rapport :

$$
\frac{\mathrm{HT}-\mathrm{H}_{1} \mathrm{~b}}{\mathrm{HT}}
$$

où $\mathrm{H}_{1} \mathrm{~b}$ est la hauteur d'insertion de la première branche.

Nous avons déterminé avec certitude l'essence pour 15 des 18 chênes : il s'agit de 15 chênes sessiles (Quercus petraea liebl.); les 3 autres, proches du sessile, ne présentaient pas tous les caractères d'identification les plus immédiats. 
L'étude de la répartition des arbres en fonction de l'âge et de l'indice de productivité de la station dont ils proviennent ne révèle aucun biais. De plus, l'indice de productivité moyen pondéré par le nombre d'arbres abattus sur chaque station vaut 1,011 , il est donc très proche de l'indice de référence 1,000 qui caractérise la courbe " hauteur dominante $=\mathrm{f}(\mathrm{AGE})$ » issue de la table de production de PArde (1962).

- De très nombreuses mesures ont été effectuées sur chaque individu. Elles ont permis, entre autres choses, de construire des tarifs donnant une estimation de la biomasse des branches, du houppier et de l'arbre entier jusqu'aux découpes fixées (BISCH, 1985).

Connaissant alors la répartition de la biomasse par classe de découpe et le volume bois fort total (tige + branches) de chaque individu, nous avons constaté l'existence de relations linéaires entre ce volume $(\mathrm{VBF})$ et tout ou partie de la biomasse de l'arbre.

Quatre tarifs de la forme Biomasse $=a+b$ VBF ont été construits par régression linéaire pondérée. On rappelle que le volume bois fort est le volume des bois de découpe supérieure à $22 \mathrm{~cm}$ de circonférence.

$$
\begin{aligned}
& \text { tarif (1): } B M T=a_{1}+b_{1} \text { VBF (biomasse totale) ; } \\
& \text { tarif (2) : } B M H=a_{2}+b_{2} \text { VBF (biomasse du houppier) ; } \\
& \text { tarif (3) : } B I 70=a_{3}+b_{3} \text { VBF (biomasse des bois de diamètre inférieur à } 7 \mathrm{~cm} \text { ) ; } \\
& \text { tarif (4) : } B I 40=a_{4}+b_{4} \text { VBF (biomasse des bois de diamètre inférieur à } 4 \mathrm{~cm} \text { ). }
\end{aligned}
$$

Le houppier est ici défini par l'ensemble des branches et l'extrémité de la tige principale au-delà de la découpe bois fort.

- La table de production du chêne dans le secteur ligérien (PARDE, 1962) donne les productions en volume bois fort total des forêts de chêne sessile traité en futaie sur station de productivité moyenne (indice de productivité $=$ indice de référence $=1,000$ ).

Sa conversion en tables de production en biomasse repose sur l'hypothèse du maintien d'une même sylviculture dans les mêmes conditions stationnelles : les données dendrométriques ne sont pas modifiées. Les seules transformations consistent à convertir les volumes $\left(\mathrm{m}^{3}\right)$ en terme de biomasse $(\mathrm{kg})$ selon les étapes suivantes :

- Pour les quatre tables, la biomasse disponible du peuplement après éclaircie et la biomasse prélevée en éclaircie à un âge donné sont respectivement estimées par :

$$
\operatorname{BDP}(k)=N 1(k) \times B 1 g(k) \text { avec B1g }(k)=a+b \frac{V 1(k)}{N 1(k)}
$$

$$
\text { et } \quad \mathrm{BE} \quad(\mathrm{k})=\mathrm{N} 2(\mathrm{k}) \times \mathrm{B} 2 \mathrm{~g}(\mathrm{k}) \text { avec B2g }(\mathrm{k})=\mathrm{a}+\mathrm{b} \frac{\mathrm{V} 2(\mathrm{k})}{\mathrm{N} 2(\mathrm{k})}
$$

avec :

$\mathrm{k}$ : numéro de ligne de la table de Pardé (varie de $\mathrm{i}=1$ à $\mathrm{i}=27$ ) (à $\mathrm{k}$ fixé correspond un âge donné) ;

N1 : nombre de tiges du peuplement après éclaircie ;

$\mathrm{V} 1$ : volume bois fort du peuplement après éclaircie ;

N2 : nombre de tiges enlevées en éclaircie ;

V2 : volume bois fort enlevé en éclaircie ;

fournis par la table de Pardé

B1g : biomasse estimée de l'arbre moyen du peuplement après éclaircie ;

$\mathrm{B} 2 \mathrm{~g}$ : biomasse estimée de l'arbre moyen enlevé en éclaircie. 
- La biomasse totale cumulée enlevée en éclaircie et la production totale à un âge donné sont alors aisément calculées. Pour une ligne $\mathrm{k}$ de la table, nous avons respectivement :

$\operatorname{BTE}(k)=\sum_{i=1}^{k} \operatorname{BE}(i)$

et $\operatorname{PT}(k)=$ BTE $(k)+$ BDP $(k)$

- Le pourcentage de biomasse enlevée en éclaircie (p. $100 \mathrm{EE}$ ), l'accroissement courant (A.c) et l'accroissement moyen (A.m) ne sont calculés que pour la table de production en biomasse totale.

A un âge donné, nous avons:

p. $100 \mathrm{EE}(\mathrm{k})=\frac{\mathrm{BTE}(\mathrm{k})}{\mathrm{PT}(\mathrm{k})} \times 100$

A.c $(k)=\frac{\text { PT }(k)-\operatorname{PT}(k-1)}{\operatorname{AGE}(k)-\operatorname{AGE}(k-1)}$

A.m $(k)=\frac{\text { PT }(k)}{\operatorname{AGE}(k)}$

\section{Résultats}

3.1. Les tarifs reienus

\subsection{Présentation}

Les quatre tarifs adoptés pour l'estimation de ${ }^{\prime}$ biomasse en fonction du volume bois fort de l'arbre sont les suivants (biomasse en $\mathrm{kg}$. VBF en $\mathrm{dm}^{3}$ ) :

\begin{tabular}{|c|c|}
\hline & $\begin{array}{c}\text { Coefficient } \\
\text { de corrélation } R\end{array}$ \\
\hline (1) $\mathrm{BMT}=8,73+0,6498 \mathrm{VBF}$. & 0,995 \\
\hline (2) $\mathrm{BMH}=-1,31+0,1085 \mathrm{VBF}$ & 0.966 \\
\hline (3) $\mathrm{BI70}=7,61+0,0606 \mathrm{VBF} \ldots \ldots \ldots$ & 0,787 \\
\hline 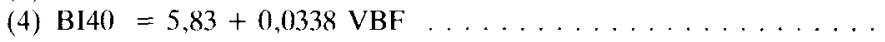 & 0.721 \\
\hline
\end{tabular}

\subsection{Remarques}

- La pondération adoptée pour les tarifs (2), (3) et (4) est en : $\frac{1}{\mathrm{VBF}}$

(la variance résiduelle du tarif non pondéré est proportionnelle à VBF). 
Le tarif (1) est pondéré en : $\frac{1}{V B F^{1.4}}$

L'ordonnée à l'origine ainsi obtenue est proche de la valeur supposée du poids anhydre total d'un brin de futaie de diamètre à la base de $7 \mathrm{~cm}$. Le nuage des résidus réduits ne présente pas de structure particulière et est peu différent de celui obtenu avec une pondération en : $\frac{1}{\mathrm{VBF}}$.

- Le tarif (2) a été construit avec 16 individus. Deux arbres fourchus ont été exclus de l'échantillon; la définition du houppier que nous avons adoptée est telle que ces deux arbres se détachent nettement du nuage de points (VBF, BMH) : pour un même volume bois fort, l'arbre fourchu possède plus de biomasse dans son houppier.

- L'ordonnée à l'origine de ce même tarif est négative. L'estimation de BMH est alors négative pour les petites valeurs de $\mathrm{VBF}\left(\mathrm{VBF}<12 \mathrm{dm}^{3}\right)$, et, pour les valeurs de VBF inférieures à $190 \mathrm{dm}^{3}$, est plus petite que l'estimation de la biomasse inférieure à la découpe $7 \mathrm{~cm}$ de diamètre.

Pour éliminer cette contradition, nous avons décidé de remplacer les estimations concernées en biomasse du houppier par les estimations en biomasse des bois de découpe inférieure à $7 \mathrm{~cm}$ de diamètre. Cette substitution se justifie par le fait qu'elle concerne des arbres de circonférence à $1,30 \mathrm{~m}$ inférieure à $50 \mathrm{~cm}$ et dont le diamètre à la base des branches est dans la grande majorité des cas inférieur à $7 \mathrm{~cm}$ (d'où $\mathrm{BI} 70=\mathrm{BMH}$.

\subsection{Les tables construites}

Elles sont présentées dans les tableaux 1 à 4 .

Nous rappelons que les valeurs indiquées se rapportent toujours à l'hectare.

La hauteur dominante est la moyenne des hauteurs des 100 plus gros arbres à l'hectare. L'arbre moyen est l'arbre de surface terrière moyenne $\left(\frac{\mathrm{G}}{\mathrm{N} 1}\right.$ pour le peuplement).

\subsection{Domaine de validité et précision}

- Le domaine de validité des tables est théoriquement défini par les limites de validité des tarifs utilisés. Elles correspondent aux valeurs extrêmes de VBF prises par les arbres échantillons : $\mathrm{VBF}=0,12 \mathrm{~m}^{3}$ et $\mathrm{VBF}=2,47 \mathrm{~m}^{3}$.

Nous admettons cependant que les estimations de biomasse obtenues en dehors de ces limites sont assez proches des valeurs réelles. Ce point de vue est discuté dans le chapitre 4.

- La précision du modèle de régression a été calculée au seuil de confiance de 95 p. 100 à partir de la formule de PERrotte (1976) qui donne la variance de la somme des estimations pour une collecte d'individus n'appartenant pas à l'échantillon. 
Cette variance est définie par:

(5) $\sigma^{2}(S)=s^{2} \cdot\left[\frac{l^{2}}{\sum_{i=1}^{n} \operatorname{mi}}+\frac{1^{2}(\bar{X}-\bar{x})^{2}}{\sum_{i=1}^{n} \operatorname{mi}\left(x_{i}-\bar{x}\right)^{2}}+\sum_{i=1}^{\sum} \frac{1}{m i}\right]$

avec $\mathrm{S}$ : biomasse estimée de la collection ;

$\mathrm{s}^{2}$ : variance résiduelle du tarif ;

$\mathrm{n}$ : effectif de l'échantillon $(\mathrm{n}=16$ ou $\mathrm{n}=18)$;

$x_{i}$ : valeur de la variable explicative (VBF) pour une observation i de l'échantillon ;

$\overline{\mathrm{x}}$ : moyenne pondérée des $\mathrm{x}_{\mathrm{i}}$ de l'échantillon :

1 : effectif de la collection :

$\overline{\mathrm{X}}$ : moyenne non pondérée de la variable explicative pour les observations de la collection ;

$\mathrm{m}_{\mathrm{i}}$ : poids d'une observation $\mathrm{i}\left(\mathrm{m}_{\mathrm{i}}=\frac{1}{\mathrm{VBF}}\right.$ ou $\left.\mathrm{m}_{\mathrm{i}}=\frac{1}{\mathrm{VBF}^{1,4}}\right)$.

L'erreur relative (ER) au seuil de 5 p. 100 se déduit de $\sigma^{2}(\mathrm{~S})$ par :

$E R=\left(t_{0.05} \sqrt{\sigma^{2}(S)} / S\right) \times 100$

$\mathrm{t}_{0,0.5}$ est la valeur du $\mathrm{t}$ de Student au seuil de 5 p. 100 pour $\mathrm{n}-2$ degrés de liberté.

Le tableau 5 fournit, pour les 4 tables de production en biomasse, les erreurs calculées sur :

- la biomasse sur pied

- la biomasse enlevée à chaque éclaircie (E.2)

- la production totale

Remarques:

1. Ces erreurs n'ont qu'une valeur théorique. leur calcul suppose implicitement qu'il n'y a pas erreur sur les volumes bois fort fournis par la table de Pardé : ce sont les erreurs calculées sur des modèles (de production).

2. Dans le cas de la biomasse sur pied ou de la biomasse enlevée à chaque éclaircie, la collection d'individus est une collection d'individus tous identiques, de volume bois fort égal au volume bois fort de l'arbre moyen.

3. Nous avons indiqué dans le tableau 5 les limites de validité théoriques d'utilisation des tarifs.

4. Pour la biomasse du houppier, les 5 premières valeurs de E1 et les 7 premières valeurs de E2 sont celles calculées sur les estimations de biomasse de découpe $\mathrm{D}<7 \mathrm{~cm}$, ces dernières se substituant aux estimations correspondantes dans la table 2 . Les erreurs sur la production totale sont calculées; avec les données du tarif (2) et les valeurs de biomasse indiquées dans cette même table.

\subsection{Relations entre la table 1 et la table 2}

L'étude de la répartition de la biomasse dans l'arbre (BISCH, 1985) a montré que la proportion de biomasse dans le houppier est linéairement indépendante de l'âge $(R=-0,010)$ et de $C 130(R=0,342)$. Elle vaut 16 p. 100 


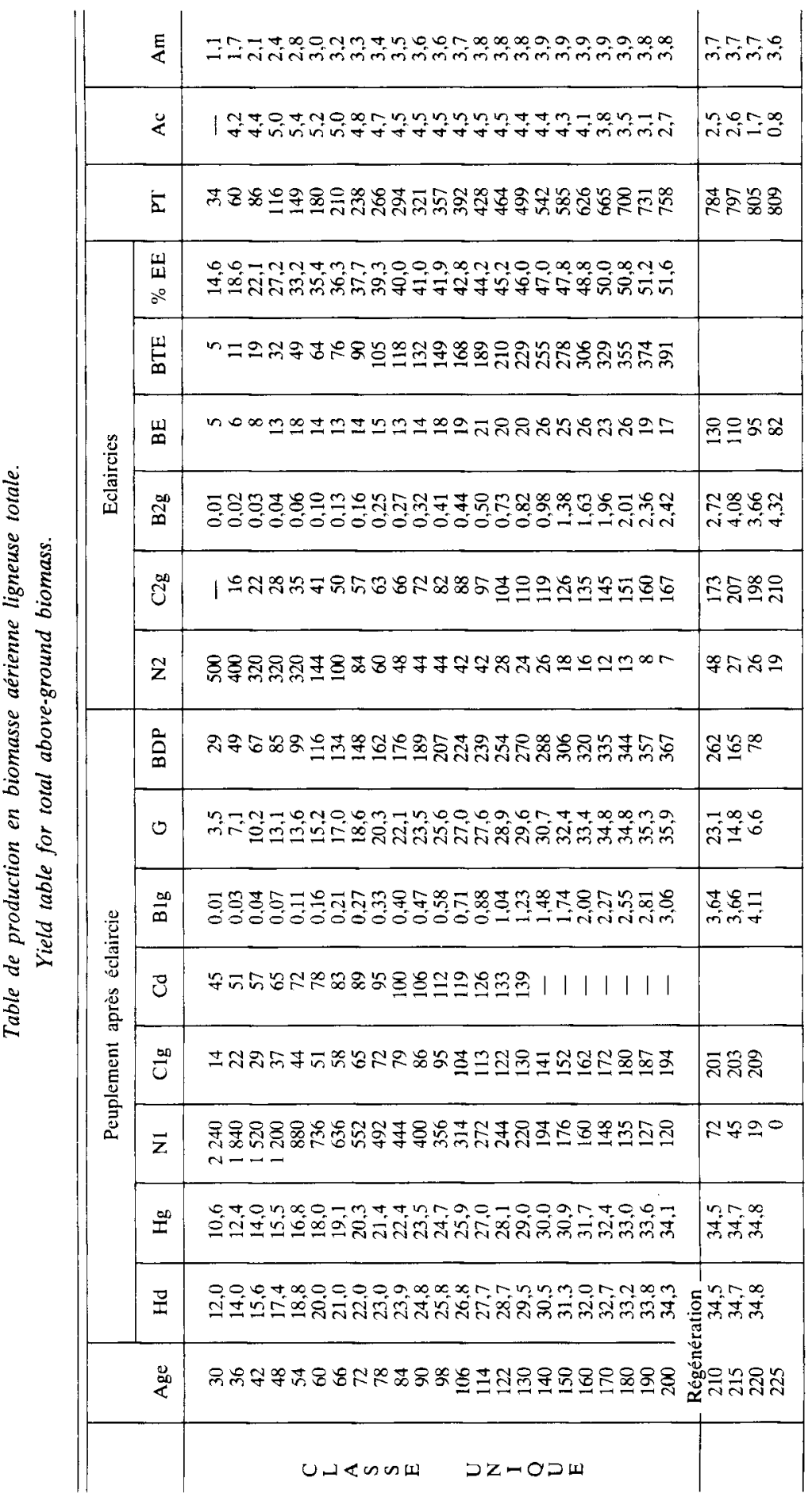

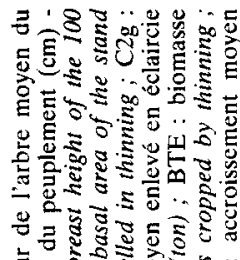

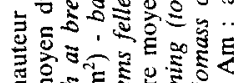

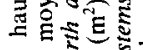

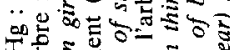

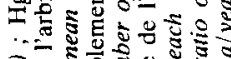

है

ช

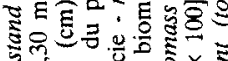

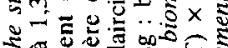

约

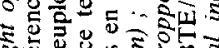

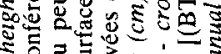

․․ำ

S. 互U

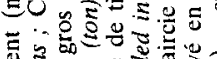

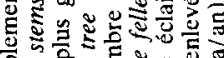

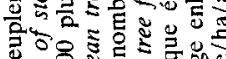

施

ว

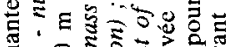

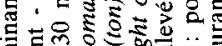

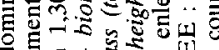

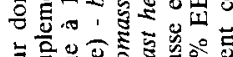

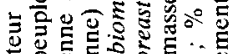

范荡

$\because$ E

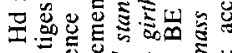

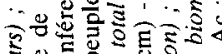

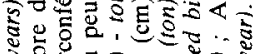

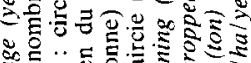

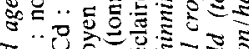

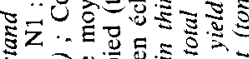

क

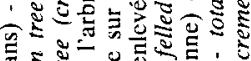

ฐ ฐ

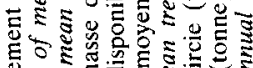

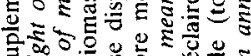

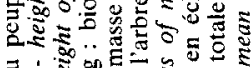

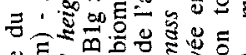

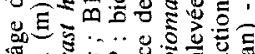

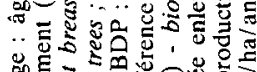
\& 8

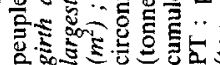




\begin{tabular}{|c|c|c|c|c|}
\hline & & $\bar{c}$ & 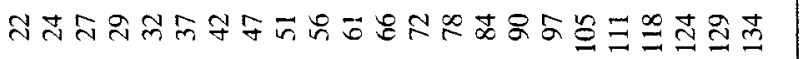 & $\stackrel{\overbrace = g}{g}$ \\
\hline & & $\stackrel{\mu}{H}$ & 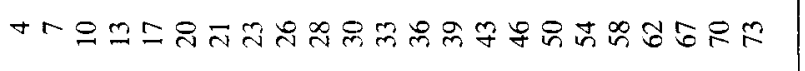 & \\
\hline & & 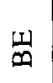 & 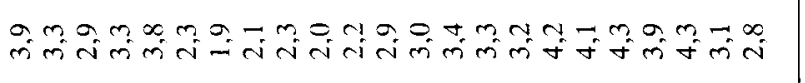 & 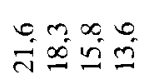 \\
\hline & $\frac{\mathscr{U}}{0}$ & $\check{\infty}^{\infty}$ & 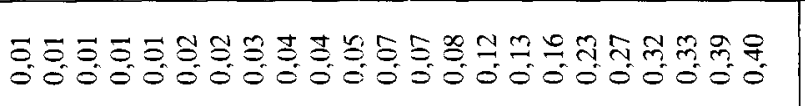 & 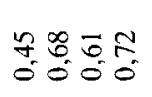 \\
\hline & & లు & 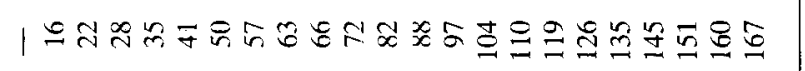 & 옹요 \\
\hline & & $\tilde{z}$ & 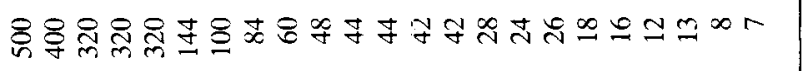 & $\infty$ 疋只 \\
\hline & & $\frac{\partial}{\Delta}$ & 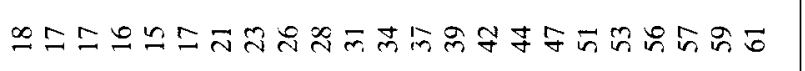 & $\forall$ 产 \\
\hline 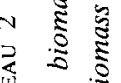 & & 0 & 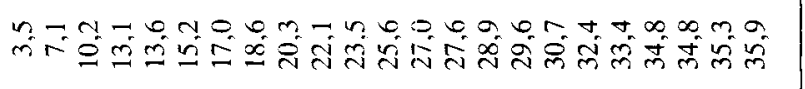 & $\begin{array}{l}-\infty \\
\dot{\gamma} \\
\dot{y}\end{array}$ \\
\hline 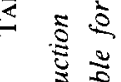 &. & $\frac{\infty}{\infty}$ & 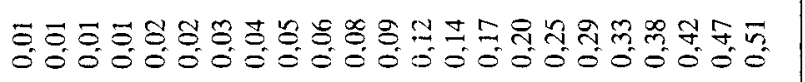 & $\begin{array}{l}8=\infty \\
8=0 \\
0=0\end{array}$ \\
\hline & 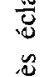 & 8 & 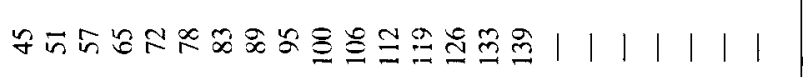 & \\
\hline$N$ & 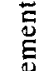 & $\frac{\infty}{U}$ & 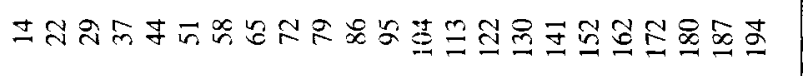 & 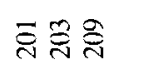 \\
\hline & ڤై & $\bar{z}$ & 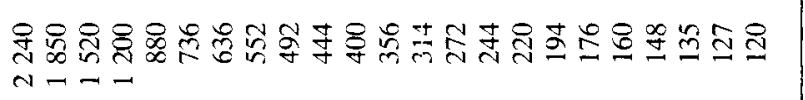 & № \\
\hline & & $\stackrel{\infty}{=}$ & 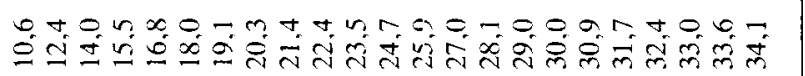 & 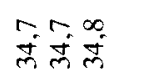 \\
\hline & & 栗 & 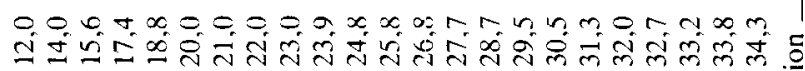 & $\begin{array}{l}r_{1}=\infty \\
\dot{m}=\dot{m}\end{array}$ \\
\hline & & $\stackrel{\infty}{<}$ & 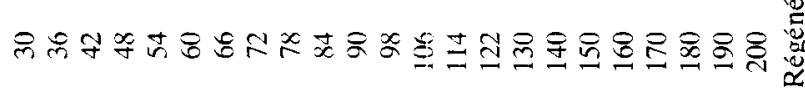 & $\underset{\sim}{\vec{N}} \underset{\widetilde{N}}{\mathbb{N}}$ \\
\hline & & & $0\lrcorner<n n$ & \\
\hline
\end{tabular}




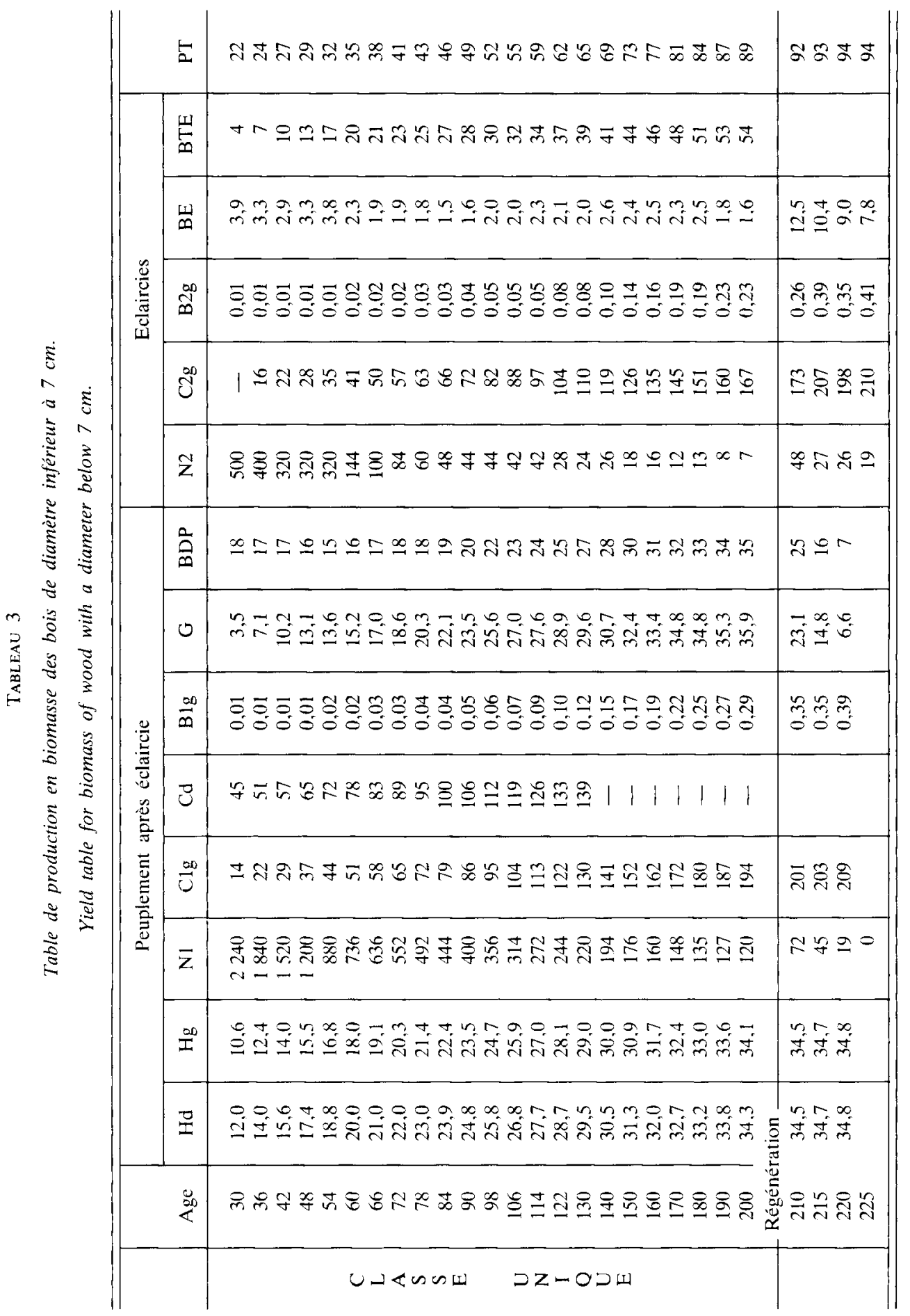




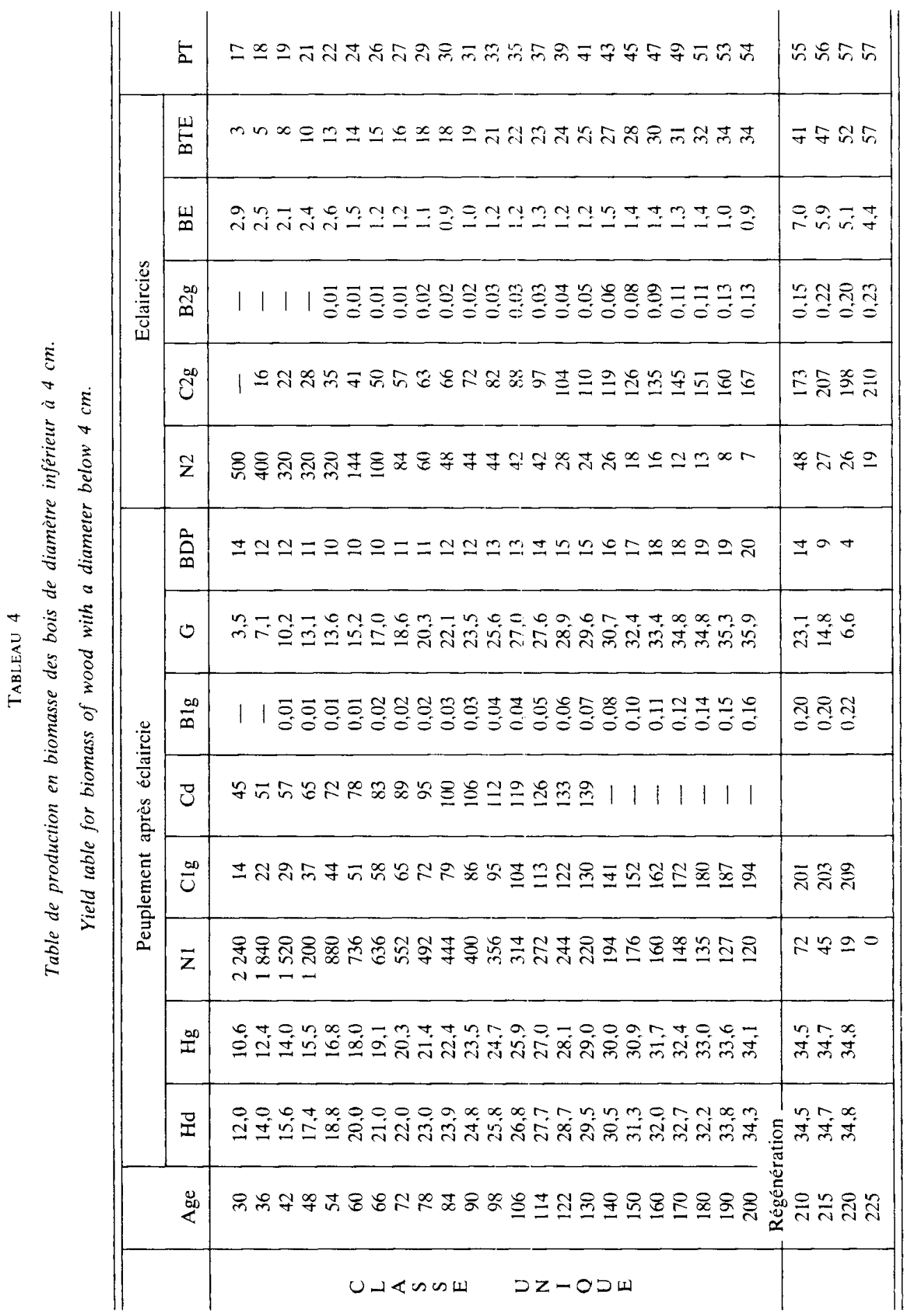




\begin{tabular}{|c|c|c|c|c|c|c|}
\hline \multirow{3}{*}{ 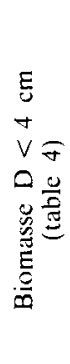 } & & $\begin{array}{l}0 x a r \\
\dot{v} \tilde{a} \tilde{y}\end{array}$ & $\stackrel{9}{\Xi}$ & 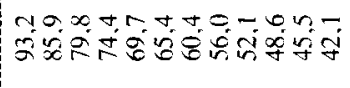 & $\vec{m}$ & 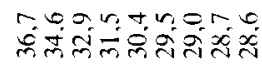 \\
\hline & 嵒 & 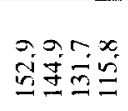 & $\bar{\Xi}$ & 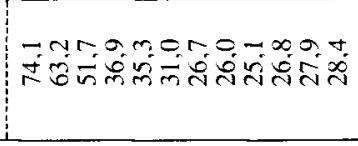 & $\frac{n}{m}$ & 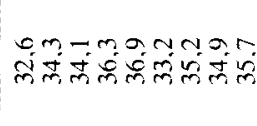 \\
\hline & $\overrightarrow{11}$ & 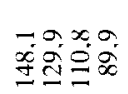 & $\ddot{8}$ & 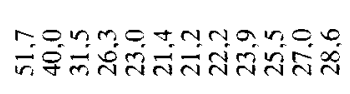 & aे & 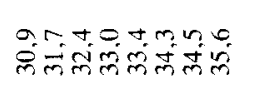 \\
\hline \multirow{3}{*}{ 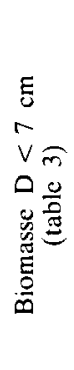 } & 章 & 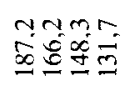 & $\stackrel{\circ}{\underline{0}}$ & 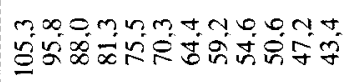 & $\vec{q}$ & 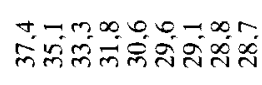 \\
\hline & 펌 & 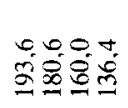 & $\stackrel{a}{=}$ & 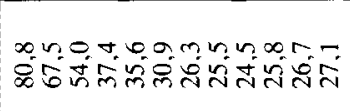 & $\ddot{\bar{i}}$ & 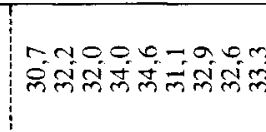 \\
\hline & 可 & $\begin{array}{l}x m=0 \\
\infty \\
\infty\end{array}$ & $\stackrel{n}{n}$ & 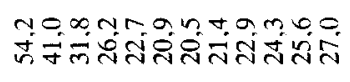 & $\vec{i}$ & 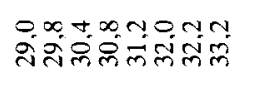 \\
\hline \multirow{3}{*}{ 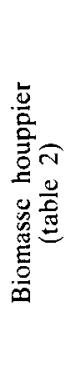 } & $\hat{y}$ & 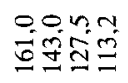 & $\stackrel{\circ}{8}$ & 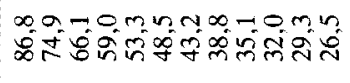 & 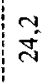 & 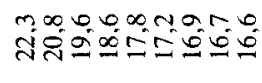 \\
\hline & Iี & 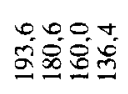 & $\stackrel{a}{=}$ & 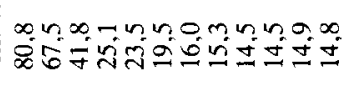 & $\ddot{g}$ & 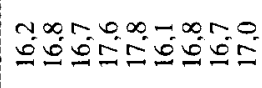 \\
\hline & 피 & $\begin{array}{l}\text { xmmo } \\
\text { कू⿻日禸 }\end{array}$ & in & 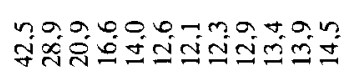 & $\stackrel{9}{ \pm}$ & 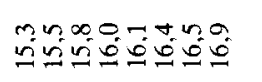 \\
\hline \multirow{3}{*}{ 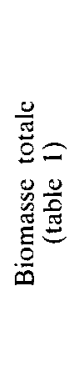 } & 3 & 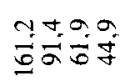 & $\stackrel{m}{m}$ & 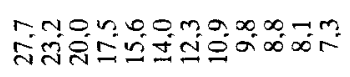 & $\begin{array}{l}0 \\
0\end{array}$ & 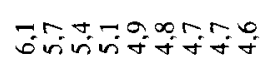 \\
\hline & $\tilde{\Psi}$ & 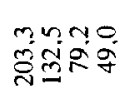 & $\stackrel{m}{m}$ & 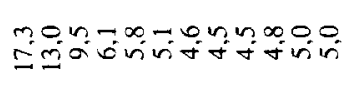 & $\dot{v i}$ & 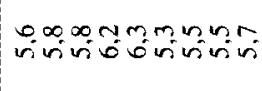 \\
\hline & 可 & $\begin{array}{l}\text { aron } \\
\text { vidiñ }\end{array}$ & $\stackrel{g}{ \pm}$ & 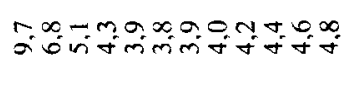 & $\stackrel{q}{\forall}$ & 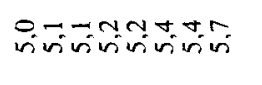 \\
\hline & $\stackrel{0}{\&}$ & ల్లిలో ํㅜㅇ & 芩 & 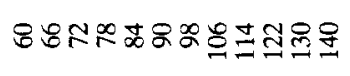 & 号 & 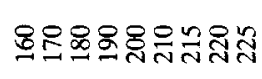 \\
\hline
\end{tabular}


Nous constatons effectivement, pour une circonférence ( $\mathrm{Clg}$ ou $\mathrm{C} 2 \mathrm{~g}$ ) de l'arbre moyen supérieure à $40 \mathrm{~cm}$, que nous obtenons une bonne estimation des valeurs de biomasse disponible sur pied (BDP) et de la biomasse enlevée en éclaircie (BE) proposées dans la table 2 par les retations: $\mathrm{BDP}_{2}=0,16 \times \mathrm{BDP}_{1}$ et $\mathrm{BE}_{2}=0,16 \times \mathrm{BE}_{1}$.

Les indices 1 et 2 correspondent aux numéros des tables.

\section{Discussion}

L'utilisation des 4 tarifs en dehors de leur limites théoriques de validité fournit des estimations de biomasse qui peuvent être discutées.

Les valeurs estimées en deçà de la limite inférieure de validité (VBF $<0,12 \mathbf{m}^{3}$ ) nous paraissent très vraisemblables en raison de la valeur des termes constants des tarifs (1), (3) et (4). Ces termes constants semblent être de bonnes estimations de la biomasse réelle quand VBF tend vers la valeur zéro.

En effet, un tarif établi par Bruciamacchie (1982) permet d'estimer à $8,27 \mathrm{~kg}$ la biomasse d'un brin de futaie dont $\mathrm{C} 130=17 \mathrm{~cm}$. (On suppose alors que la circonférence à la base vaut $22 \mathrm{~cm}$ ). Cet auteur fournit également des estimations de biomasse sur pied pour des peuplements dont la hauteur moyenne varie entre 3 et $13 \mathrm{~m}$. Pour les hauteurs supérieures à $10 \mathrm{~m}$, elles sont plus élevées que celles que nous proposons du fait d’une densité plus forte que celle préconisée par la table de Pardé.

Nous ne possédons pas d'éléments de comperaison pour les valeurs estimées de biomasse au-delà de la limite de validité supérieure des tarifs (VBF $\left.>2,5 \mathrm{~m}^{3}\right)$. Cependant, nous avons montré par ailleurs que la proportion en biomasse du houppier était indépendante de l'âge entre 50 et 140 ans, alors qu'en taillis-sous-futaie, cette même proportion augmente avec l'âge (BiscH, 1985). Nous expliquons cette différence par le contrôle continu qu'exerce le sylviculteur sur la croissance de l'arbre de futaie : le houppier est contraint à se développer dans un espace limité. Ces contraintes étant maintenues pendant toute la durée de vie de l'arore, il paraît raisonnable d'admettre que la répartition de la biomasse dans l'arbre n'est pas fondamentalement modifiée audelà de 140 ans, et par conséquent que les relations linéaires établies demeurent correctes au-delà de cette limite d'âge imposée par l'échantillon.

De même que l'utilisation des tarifs, le calcul des erreurs relatives n'est en théorie réalisable que dans la zone de validité définie par les valeurs extrêmes des variables mesurées de l'échantillon. Les erreurs présentées clans le tableau 5 en dehors de cette zone n'ont qu'une valeur indicative. Dans la partie supérieure du tableau, les erreurs sur la biomasse disponible sur pied et sur la biomasse prélevée en éclaircie sont très élevées. Ceci s'explique par la valeur élevée de l'effectif 1 de la collection et par l'éloignement de ces individus par rapport à la moyenne pondérée de l'échantillon : $\mathrm{X}-\mathrm{x}$ est grand [cf. équation (5)].

Les erreurs sur la production totale sont également élevées : elles tiennent implicitement compte des erreurs calculées sur la bionasse prélevée en éclaircie avant 55 ans.

Ces observations ne remettent pas en cause la formule établie par Perrotte (1976). 
mais révèlent les faiblesses de l'échantillon qui, à l'origine, a été conçu pour d'autres travaux que ceux décrits ici (Bısch, 1985).

Rappelons enfin que toutes les erreurs annoncées dans le tableau 5 résultent d'un calcul qui ne prend pas en compte les erreurs sur les volumes bois fort fournis par la table de production Pardé; en effet, nous ne connaissons pas la précision de cette table.

Les erreurs annoncées sont donc inférieures aux erreurs réelles: ce sont des erreurs théoriques inhérentes au modèle.

Par ailleurs, il est important de souligner d'un point de vue fondamental que la production totale et les accroissements moyens sont sous-estimés : une bonne partie de la biomasse produite avant 30 ans n'est pas prise en compte. Pour des raisons techniques évidentes, la récolte de la biomasse au cours des dégagements et nettoiements est actuellement peu envisageable. De plus, il est difficile de la chiffrer : elle dépend de la densité du peuplement, du nombre, de la date et de l'intensité des interventions.

Par contre, la récolte de la biomasse au cours des cloisonnements n'est pas impossible. Dans une régénération de hauteur moyenne $4,5 \mathrm{~m}$, de densité 35700 tiges/ ha, Bruciamacchie (1982) estime que la biomasse susceptible d'être récupérée avec un cloisonnement $50 \mathrm{~m} \times 50 \mathrm{~m}$, de $3 \mathrm{~m}$ de large, est de l'ordre de 4,3 tonnes/ha.

Enfin, il convient de rappeler que les tables proposées ici, comme la table de Pardé, ne tiennent pas compte de la biomasse produite par les essences d'accompagnement du chêne et en particulier le hêtre.

\section{Conclusion}

L'intérêt de ces tables est différent selon qu'il s'agit de la table de production en biomasse totale, de la biomasse du houppier ou des découpes inférieures à 7 et $4 \mathrm{~cm}$ de diamètre. (Biomasse foliaire exclue dans tous les cas).

La première offre la possibilité de comparer la production du chêne traité en futaie à celle d'autres traitements ou à la production d'autres essences.

L'accroissement courant maximal est atteint vers 55 ans : il est estimé à 5,4 tonnes/ ha/an. L'accroissement moyen maximal est atteint vers 160 ans et vaut 3,91 $\pm 0,24$ tonnes/ha/an (au seuil d'erreur de 5 p. 100). Par comparaison, les productions moyennes des taillis simples de chênes, charme, bouleau, en peuplement pur ou mélangé, âgés de 20 à 40 ans et situés en conditions écologiques différentes varient de 1,9 à 4,1 tonnes/ha/an (Kestemont, 1970 ; Auclair \& Metayer, 1980 ; Ranger et al., 1981 ; Bouchon et al., 1985). Ces productions moyennes ne correspondent sans doute pas aux productions moyennes maximales : pour le robinier dans le Val-de-Loire, celleci est atteinte vers 9 ans et est de l'ordre de 6 tonnes/ha/an (PAGÈs, 1985).

En outre, cette première table permet d'estimer rapidement la biomasse disponible sur pied de futaies de chênes d'àge varié, alors que les estimations rencontrées dans la bibliographie concernent généralement des peuplements d'un âge donné. 
L'intérêt des tables de production du houppier, des bois de diamètre inférieur à $7 \mathrm{~cm}$ (découpe bois fort) et de diamètre inférieur à $4 \mathrm{~cm}$ réside dans l'estimation de la biomasse susceptible d'être récoltée à chaque éclaircie. L'utilisation de ce potentiel énergétique dans les années à venir n'est pas forcément une utopie : la production de méthanol par transformation thermochimique de la biomasse forestière est à l'ordre du jour (Anonyme, 1985).

Dans cette perspective, il faut noter que les données de l'Inventaire Forestier National pourront permettre l'évaluation de ce potentiel dans la région Centre par conversion des volumes bois fort des futaies de chêne sessile en terme de biomasse.

Reçu le 28 mars 1986.

Accepté le 28 novembre 1986.

\section{Remerciements:}

Ce travail n’aurait pu être mené à bien sans les autorisations d’abattage accordées par la Direction Régionale Centre de l'Office National des Forêts et sans la collaboration financière de sa Direction technique.

Nos remerciements vont aussi à M. BRETon qui a autorisé le prélèvement de quelques tiges en forêt privée de Marchenoir.

Enfin, je tiens à remercier toutes les personnes avec: lesquelles nous avons effectué le travail de terrain: Christine Haffner, Pierre Forssac et l'équipe technique de la Station de Sylviculture d'Orléans: Alain Moreau, Jean-Marie Vallét et Paul Romary.

\section{Summary \\ An example of conversion from volume yield able into biomass yield tables: oak in central France.}

The yield table for oak high forests in the Center of France, proposed by PARDÉ in 1962, is converted into four biomass yield tables:

- a yield table for total above-ground biomass (tabie 1);

- a biomass yield table for the crown (table 2);

- a biomass yield table for wood with a diameter helow $7 \mathrm{~cm}$ (table 3) ;

- a biomass yield table for wood with a diameter below $4 \mathrm{~cm}$ (table 4 ).

In each case, the foliar biomass is not taken into account.

The conversion is computed with regressions of the form : Biomass $=a+b$ VBF established with a sample of 18 trees correctly selected in oak high forests of the Center of France. VBF is the volume of wood with a diameter larger than $7 \mathrm{~cm}$. 
These regressions are used to convert into biomass the VBF of the mean tree felled in thinning or remaining in the stand after thinning.

We give a theoretical statistical error due to the model on the estimation of the stand biomass after thinning (BDP $\rightarrow E 1)$, the biomass felled in each thinning $(B E \rightarrow E 2)$ and the total yield $(\mathrm{PT} \rightarrow \mathrm{E} 3)$.

Thus, the maximal mean annual increment is estimated at $3,91 \pm 0,24$ tons/ha/year.

A very simple relationship is established between table 1 and table 2 .

We discuss the precision and the validity of the tables and the practical importance of the different established tables.

Key words: Biomass, yield table, Quercus petraea liebl, high forest, regression, error.

\section{Références bibliographiques}

Anonyme, 1985. L'intérêt d'un programme méthanol. Biomasse actual. $\mathrm{n}^{\circ} 40,10-11$

Auclair D., Metayer S., 1980. Méthodologie de l'évaluation de la biomasse aérienne sur pied et de la production en biomasse des taillis. Acta Ecologica, Ecol. Appl., 1, 357-377.

Bisch J.L., 1985. Influence du traitement sylvicole (futaie et taillis-sous-futaie) sur la répartition de la biomasse dans le chêne en région Centre. INRA, Station de Sylviculture, Orléans, 85/42, $26 \mathrm{p}$.

Bisch J.L., Auclair D., 1987. Influence of the silvicultural treatment (coppice with standards, high forest) on oak aboveground biomass distribution in Central France. Forestry (soumis pour publication).

Bouchon J., Nys C., Ranger J., 1985. Cubage, biomasse et minéralomasse : comparaison de trois taillis simples des Ardennes primaires. Acta Ecologica, (Ecol. Plant., 6 (20), 1, 53-72.

Bruciamacchie M., 1982. Structure, croissance et biomasse des régénérations naturelles de chêne rouvre (Quercus petraea liebl.). E.N.I.T.E.F., Nogent-sur-Vernisson; Mémoire de fin d'études. INRA, station de Sylviculture, Nancy, 82/02, 45 p.

Cannel M.G.R., 1982. World Forest Biomass and Primary Production Data. 391 p., Academic press, London.

Kestemont P., 1970. Etude de la biomasse et de la productivité de trois peuplements en taillis simple. Bull. Inst. r. Sci. nat. Belg., 46 (17), 14 p.

LE Goff N., 1984. Indice de productivité des taillis-sous-futaie de chêne dans la région Centre. Ann. Sci. For., 41 (1), 1-34.

O.C.D.E., 1984. La biomasse source d'énergie. O.C.D.E., Paris, 145 p.

PAGÈs L., 1985. Les taillis de robinier du Val-de-Loire. Croissance, biomasse, régénération. Thèse, spécialité Ecologie Végétale, Université de Paris-Sud (Orsay), 74 p.

Pardé J., 1962. Table de production pour les forêts de chêne rouvre de qualité tranchage du secteur ligérien. Notes Techiques Forestières, Station de recherches et expériences forestières, Nancy, $\mathrm{n}^{\circ} 11,6 \mathrm{p}$.

Pardé J., 1977. Biomasses forestières et utilisation totale des arbres. Rev. For. Fr., 29 (5), $333-$ 342.

Perrotre G., 1976. Théorie de la régression linéaire multiple pondérée en vue de l'application aux calculs des tarifs de cubage et des tables de production. Session de formation continue sur les tarifs de cubage et tables de production, E.N.G.R.E.F., Nancy, 14 p.

Ranger J., Nys C., Ranger D., 1981. Etude comparative de deux écosystèmes forestiers feuillu et résineux des Ardennes primaires françaises. I - Biomasse aérienne du taillis-sous-futaie. Ann. Sci. For., 38 (2), 259-282.

Young H.E., 1974. The complete tree institute of the university of Maine. In : Meeting of A.P.A.T.A.P.P.I. committee on whole tree utilization, Convigton, Virginia, 6 p. 